\title{
CA19-9 versus CA-125 in Endometriosis
}

\author{
${ }^{1}$ Tharwa H. Hassan AL-Tai, ${ }^{2}$ Hayfaa S. AL-Hadithi, ${ }^{3}$ Hind S. Abdulsalam, \\ ${ }^{I}$ MSc Microbiology; Assistant Lecturer-Dept. of Biology/Collage of Medicine/University of Baghdad \\ ${ }^{2}$ FICM/path.; Assistant Professor-Dept. of Microbiology \& Immunology/Collage of Medicine/University of \\ Baghdad \\ ${ }^{3}$ MBChB, FIBOG, CABOG; Lecturer-Dept. of Gynecology/Collage of Medicine/University of Baghdad
}

\begin{abstract}
Summary:
Background: -Endometriosis is a condition characterized by the presence of endometrial tissue in ectopic foci outside the uterus. The lesions are not cancerous, but they can develop to the point that they cause obstruction or adhesion that attach to nearby organs, causing pain, inflammation, and sometimes infertility. Recently a tumor marker has been used in endometriosis which is CA-125 (cancer antigen or carbohydrate antigen 125). Objective:-To evaluate the affectivity of serum CA19-9versus serum CA-125in the diagnosis of endometriosis. Patients and methods: -This study applied on 51women with endometriosis compared to 33 healthy women as control group starting from November 2011 to end of January 2012.Their serum samples were collected to investigate serum concentrations of CA-125 and CA19-9 by using ELISA technique.

Results: -Serum level of CA-125 and CA19-9 weresignificantly increase inpatientswith endometriosis.Serum CA-125 level was significantly higher in stage III and IV, whileserum CA19-9 levels were only significantly increase in those with stage IV endometriosis. Alsothe highest sensitivity and PPV was for CA-125, while serum CA19-9 test had 5\% sensitivity and 95\% specificity but with lower accuracy than CA-125.

Conclusion:-Serum CA-125 and CA19-9 were significantly higher in endometriosis patients than in control group, and their levels were very useful in predicting the severity of the disease.
\end{abstract}

Keywords: Endometriosis,CA-125 and CA19-9.

\section{Introduction}

Endometriosis is a condition characterized by the presence of endometrial tissue in ectopic foci outside the uterus. Although not life threatening, endometriosis is a crippling disease that severely compromises patient's health and leads to infertility.Women with endometriosis may have chronic immune activation due to the presence of endometrial deposits[1]. The lesions are not cancerous, but they can develop to the point that they cause obstruction or adhesion that attach to nearby organs, causing pain, inflammation, and sometimes infertility[2]. It has been seen as an endocrinological condition asits continuous growth can be interrupted through hormonal manipulationin particular with oestrogen withdrawal[3]. The most commonsymptoms are dysmenorrhoea, dyspareunia and low back pain that are worse around menses[4].

Endometriosis can affect any female, from premenarche to postmenopause, regardless race, ethnicity or whether they have had children or not. It is primarily a disease of reproductive years[5]. But in women with pain, infertility, or both, the frequency is $35-50 \%[6]$.

Recently a tumor marker hasbeen used in endometriosis which is CA- 125 (cancer antigen or carbohydrate antigen 125), itis a substance that is found in greater concentration in tumor cell than in other cells of the body[7]. Many body fluids as milk, amniotic fluid, peritoneal or pleural fluid, cervical mucus and seminal fluid contain CA- 125[8]. The normal ovary does not seem to be an important source of CA-125 production[9]. However primary cultures of human ovarian surface epithelial cells secret detectable but lowest concentration of CA-125[10]. A number of benign conditions can cause elevations of the CA-125 level, including pregnancy, endometriosis, uterine fibroids (benign tumors), pancreatitis, normal menstruation, pelvic inflammatory disease, and liver disease [11\&12].

Another tumor marker is Cancer antigen 19-9 (CA19-9) which is a protein that exists on the surface of certain cancer cells. Although CA19-9 shaded from the tumor cells, it does not cause cancer, it is only useful to follow the course of cancer[13]. CA19-9 is synthesized by normal cell in pancreatic and bile ducts, bronchial, salivary gland and endometrium. Secretion from these organs are rich in CA19-9, but very little antigen appears in the blood of healthy individuals [14].

\section{Materials and method}

A study was conducted in the period between November 2011 and January 2012. Patients selected from those who attend "Kamal Al-Samari" hospital and Baghdad medical city teaching hospital as51 newly 
diagnoseduntreated cases of endometriosis and 33 apparently healthy fertile women without endometriosis their age was matched with the patients group ranging from (18-45) years.

Blood samples were obtained from each individual by venous puncture, then was left to clot at room temperature, centrifuged and serum was collected for the detection of human CA125 andCA19-9by using Enzyme linked immunosorbent assay (ELISA) technique.

These are measured through the use of CA-125 EILSAkit (Human-52050) and CA19-9 EILSA kit (Human-52060). The Cut-Off points were adjusted for CA-125 and CA 19-9 as normal serum level must be <35 $\mathrm{u} / \mathrm{ml}$ and $<40 \mathrm{u} / \mathrm{ml}$ respectively.

Data expressed as mean \pm SD. Statistical differences between the groups were determined according to ANOVA test and student t-test the results were considered significant when pvalue $<0.05$.

\section{Results}

Table (1) shows the significant increase of serum level of CA-125 and CA19-9 inpatientswith endometriosis.

Table (1): Distribution of the serum CA-125 and CA 19-9

\begin{tabular}{|cc|c|c|c|}
\hline & Type & $\mathrm{N}^{*}$ & Mean \pm SD & p. value \\
\hline \multirow{2}{*}{ CA-125 } & Patient & 51 & $22.367 \pm 4.587$ & $9.167 \pm 2.952$ \\
& Control & 33 & $17.406 \pm 8.384$ & $0.008 * *$ \\
\hline \multirow{2}{*}{ CA19-9 } & Patient & 51 & $10.574 \pm 6.239$ & $0.025^{* * *}$ \\
& Control & 33 & $* * *$ significant $=\mathrm{P}<0.05$, & \\
\hline
\end{tabular}

Endometriosis was classified into 4 stages according to the revised American Fertility society, but in our study there was no patient atstage I. The levels of all measured markers in relation to the stage of the disease were shown in table (2).

Table (2): Seroimmunological levels distributed according stages of endometriosis

\begin{tabular}{|lc|c|c|c|}
\hline \multicolumn{1}{|l|}{ Stage } & $\mathrm{N}$ & Mean $\pm \mathrm{SD}$ & Range \\
\hline \multirow{4}{*}{ CA-125 } & 2.00 & 2 & $18.333 \pm 10.546$ & $(10.88-25.79)$ \\
& 3.00 & 32 & $23.184 \pm 20.818$ & $(3.68-172.28)$ \\
& 4.00 & 17 & $21.305 \pm 16.448$ & $(5.14-70.09)$ \\
\hline \multirow{3}{*}{ CA19-9 } & 2.00 & 2 & 14.480 & $(14.48-14.48)$ \\
& 3.00 & 32 & $14.091 \pm 14.000$ & $(0.28-79.84)$ \\
& 4.00 & 17 & $17.929 \pm 11.489$ & $(4.08-45.46)$ \\
\hline
\end{tabular}

Figure (1) shows that serum CA-125 level was significantly higher in stage III and IV, while figure (2) shows that serum CA19-9 levels were only significantly increase in those with stage IV endometriosis.

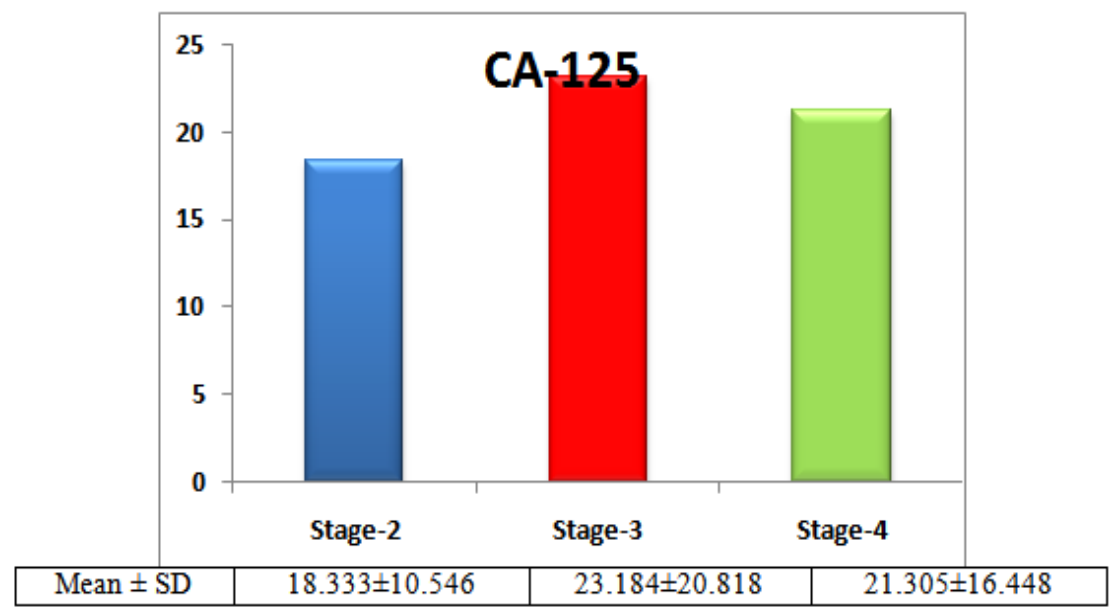

Figure (1): Serum CA-125 level in different stages of endometriosis 


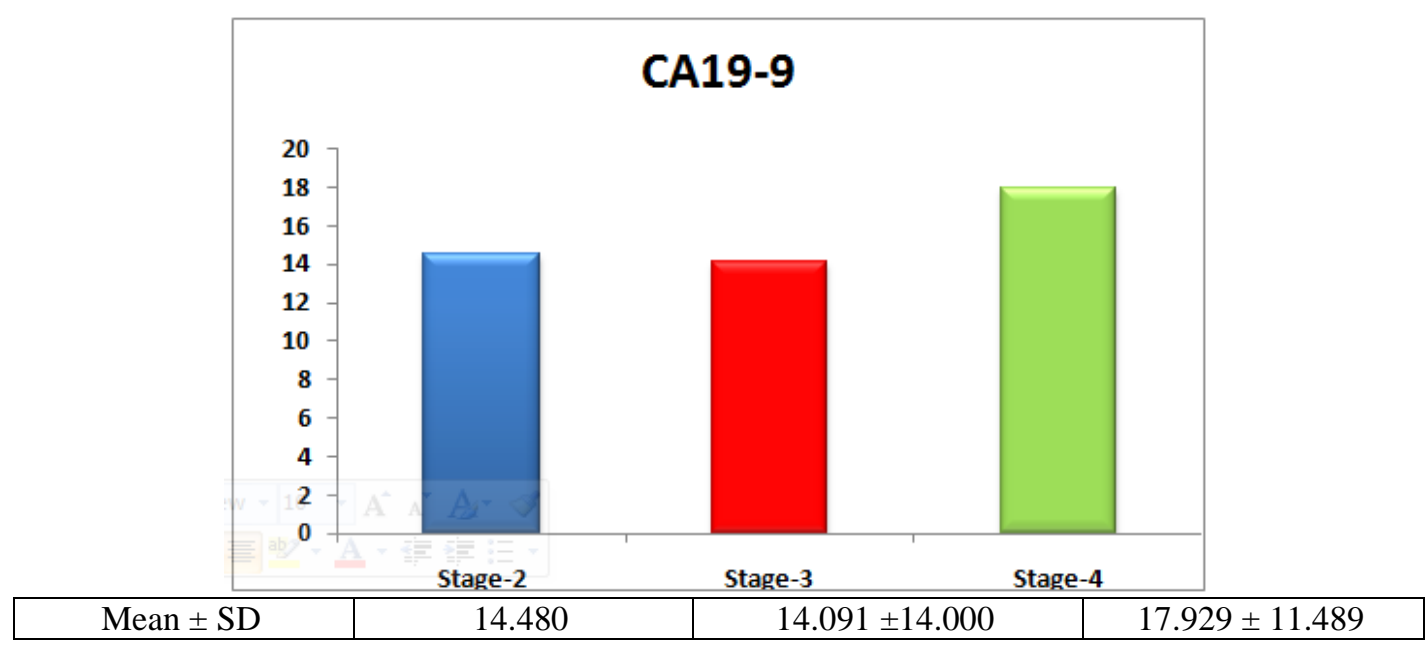

Figure (2): Serum CA19-9 level in different stages of endometriosis

Table (3) shows that the highest sensitivity and PPV was for CA-125, while serum CA19-9 test had $5 \%$ sensitivity and $95 \%$ specificity but with lower accuracy than CA-125.

Table (3): Validity parameters for the studied endometriosis in differentiating it from healthy control

\begin{tabular}{|c|c|c|c|c|c|}
\hline Overall & Sensitivity & Specificity & Accuracy & PPV* & NPV** \\
\hline CA-125 & $10 \%$ & $90 \%$ & $60.7 \%$ & $100 \%$ & $58 \%$ \\
\hline CA19-9 & $5 \%$ & $95 \%$ & $58.8 \%$ & $100 \%$ & $57 \%$ \\
\hline
\end{tabular}

\section{Discussion}

In the present study, serum level of CA-125 was significantly elevated in patient when compared with control group. This result agrees with previous studies which have been concluded that elevated CA-125 levels has been associated with a number of benign gynecologic diseases such as endometriosis, and CA-125 has been detected in several normal tissues including the endometrium and the lung[15]. The present study, shows that serum CA-125 level were significantly higher in stage III and IV, and this result agrees with many recent studies were they also found more elevated level of serum CA-125 with more advanced stages (III and IV) of endometriosis when compared to stage (I and II)[16].Vivianet alin 2006 demonstrated a higher CA-125 levels in both serum and peritoneal fluid in women with advanced pelvic endometriosis[15]. Lastly,in 2011Patrelli et al. considered serum CA-125 measurement as a consolidated method for diagnosing evenminimal or mildendometriosis because of its higher level than that in the disease free women[17]. In the present study, the mean of serum CA19-9 in endometriosis women were higher from those without endometriosis, but CA19-9 has inferior sensitivity to CA-125. These results agree with many other studies[18]. This study, showed that serum CA19-9 was significantly increase only in those with stage IV endometriosis in comparison with other stages, this result agrees with other studies which revealed that this tumor marker was positively correlated with the severity of the disease and it's level was increased in accordance with the advancement of clinical stage of endometriosis[19]. Our study concludes the highest specificity of CA-125 measures in endometriosis patient but with low sensitivity, this result agrees with some studies which focused mainly on the validity of serum CA-125 in detection of endometriosis and concluded that it could be used as an adjuvant together with other markers[20]. As our study, showed that CA19-9 has higher specificity and lower sensitivity than CA-125, several studies have been attempted to compare between CA19-9 and CA-125 measurements, most of them agreed with our results [21].Other studies documented that, tumor markers such as CA-125 and CA19-9 lack sensitivity and specificity when used to screen for endometriosis. Nevertheless, researches continue on the development of blood tests for the diagnosis of endometriosis, even yet they were available for routine use in clinical practice [22].

\section{Conclusion}

Serum CA-125 and CA19-9 were significantly higher in endometriosis patients than in control group, andtheir levels were very useful in predicting the severity of the disease. 


\section{References}

[1] Rawson JMR. (2011). Prevalence of endometriosis in asymptomatic women. J.Reprod.Med. 36: 513-515.

[2] FirmanAbdullah (2009). Endometriosis. J. Fertil. Steril. 33: 200-210.

[3] Brosens IA.(2007).Endometriosis- disease because it is characterized bybleeding. American journal of Obstetrics and Gynecology. 176:2263-267.

[4] Brosens IA.andBrosens JJ. (2009). Is laparoscopy the gold standard for the diagnosis of endometriosis? Eur. J. Obstet. Gynecol.Reprod.Biol. 88: 117-119.

[5] DharmeshKapoor and Willy Davila. (2005). Endometriosis.e Medicine. 119: 301-330.

[6] Giudice LG. and Kao LC.(2004). Endometriosis. Lancet ; 364: 1789.

[7] Osman N., O'Leary N., Mulcahy E., Barrett N., Wallis F., Hickey K. and Gupta R.(2008). Correlation of serum CA125 with stage, grade and survival of patients with endometriosis and epithelial ovarian cancer. Ir. Med. Journal. 101(8): 245-7.

[8] Meisser A., Campana A and Bischof P.(1996). CA125 in seminal plasma, correlation with serum parameters. Hum. Reprod. 11: 574-578.

[9] Michael, Herbert M., Pinedo and Umberto Veronesi.(1995). Oxford text book of Oncology . Volume 1. pp. 220-250.

[10] Zeimet AG., Offner FA., Muller-Holzner E., Widschwendter M., Abendstein B., Fuith LC., Daxenbichler G and Marth C.(1998). Peritoneal and tissues of the female reproductive tract as physiological sources of CA-125 tumor biology. 19: $275-282$.

[11] Locker GY.,Hamilton S. and Harris M.et al.(2006). Use of tumor markers in endometriosis disease. Journal of Clinical Oncology. 24: 5313-5327.

[12] Hussain and Fazal.(2009). Gynecologic tumor markers. New England journal Medicine. 13: 110-30.

[13] Thomas T. and Clayron L.(1997). Use CA19-9 tumor marker in the patient endometriosis. Philadelphia. [18th Edition]: 15-30.

[14] Duffy MJ.(2009). Review of CA19-9.Ann.Clin.Biochem.26:379-387.

[15] Vivian Ferreira do Amaral, Marcos Felipe Silva de Sa andRuialbertoFerriani et al.(2006). Positive correlation between serum CA125 and advanced endometriosis. Sao-Paulo. Med.J. Vol.-124, No. 4:151-318.

[16] Xavier P., Beirese J. and Belo L. et al.(2005). Are we employing the most effective CA-125 and CA 19-9 cut-off values to detect endometriosis? Eur. J. Obstet. Gynecol. Reprod Biol. 123: 254-255.

[17] PatrelliTS., Berretta R .andGizzo E.(2011). CA-125 serum values in surgically treated endometriosis patients and its relationship with anatomic sites of endometriosis and pregnancy rate.Fertil.Steril. 95: 393-396.

[18] Matalliotakis I., Panidis D., Vlassis G., Neonaki M., Goumenou A. andKormantakis M. et al.(2008). Unexpected increase of the CA 19-9 tumor marker in patients with endometriosis. Eur. J. Gynaecol. Oncol. 19: 498-500.

[19] Li Hui.(2010). Endometriosis radioimmunoassay CA-125 antigen CA 19-9 antigen. Journal of clinical Obstetric and Gynecology. 80: 200-11.

[20] Bedaiwy MA., Falcone T. and Sharma RK. et al.(2002). Predication of endometriosis with serum and peritoneal fluid markers: a prospective controlled trial. Human Reproduction. 17(2): 426-431.

[21] Barbieri RL.(2011). Correlation of CA-19-9. Fertil.Steril. 46: 66-80.

[22] Dogan S., Djalali S., Agic A., Diedrich K. andHornung D. et al.(2008). Diagnostic endometriosis-New tests periphereal blood. J. Gynecol. Endocrinol.18(2): 14-17. 